\title{
RESEARCH NOTES
}

\section{ON OSCULATORY INTERPOLATION BY TRIGONOMETRIC POLYNOMIALS}

\author{
D.J. NEWMAN \\ Department of Mathematics \\ Temple University \\ Philadelphia, Pennsylvania 19122 U.S.A.
}

\author{
L.A. RUBEL \\ Department of Mathematics \\ University of Illinois \\ Urbana, Illinois 61801 U.S.A.
}

(Received May 21, 1979)

ABSTRACT. A short and simple proof is given that osculatory interpolation by trigonometric polynomials is always possible.

KEY WORDS AND PHRASES. Trigonometric polynomials, interpolation, osculatory interpolation, squares of polynomials.

1980 MATHEMATICS SUBJECT CLASSIFICATION CODES. $42 \mathrm{~A} 12$

It is an elementary fact that if $2 \mathrm{n}+1$ points $\theta_{j}$ with

$$
-\pi \leq \theta_{0}<\theta_{1}<\ldots<\theta_{2 n}<\pi
$$


are given, then there exists an element $f$ of $T_{n}$, the class of trigonometric polynomials of degree $\leq \mathrm{n}$;

$$
f\left(e^{i \theta}\right)=\sum_{k=-n}^{n} a_{k} e^{i k \theta}
$$

so that $f\left(e^{i \theta_{j}}\right)=w_{j}$ for $j=0,1, \ldots, 2 n$, where $w_{0}, w_{1}, \ldots, w_{2 n}$ is any given sequence of complex numbers. A proof (in the real case) is given in Example 5 on page 38 of Davis' book [1], and on page 53, Problem 13 asks, somewhat enigmatically, "Discuss the possibility of osculatory trigonometric interpolation." In this note, we give a simple proof of a theorem that answers this problem and a bit more.

THEOREM. Given two sets of $n$ complex numbers, $w_{1}, \ldots, w_{n}$ and $w_{1}^{\prime}, \ldots, w_{n}^{\prime}$, there exists a trigonometric polynomial $f$ of the form 2 ), with $a_{0}=0$, so that $f\left(e^{i \theta_{j}}\right)=w_{j}$ and $f^{\prime}\left(e^{i \theta_{j}}\right)=w_{j}^{\prime}$ for $j=1,2, \ldots, n$.

REMARK. The osculatory case is where all the $w_{j}=0$. Our theorem amounts to letting the $\theta_{j}$ coalesce in pairs. Our proof depends on a trick that does not seem to cover more general kinds of coalescence, for which there is surely a corresponding result. The lemma we use to prove the theorem sheds a little light on the problem considered in [2-4] about the number of vanishing coefficients in the square of a polynomial.

PROOF OF THE THEOREM. Let $T_{n}^{0}$ be the subclass of $T_{n}$ where $a_{0}=0$, so that $T_{\mathrm{n}}^{0}$ is a vector space of dimension $2 \mathrm{n}$, and consider the $2 \mathrm{n}$ linear functionals consisting of point evaluations of elements of $T_{n}^{0}$ at the $e^{i \theta_{j}}$ and also of point evaluations of their first derivative at the $e^{i \theta_{j}}, j=1, \ldots, n$. By standard considerations of linear algebra it is enough to prove that these functionals are linearly independent, or equivalently, that if $f \in T_{n}^{0}$ and if $f\left(e^{i \theta} j\right)=f^{\prime}\left(e^{i \theta} j\right)=0$ for $j=1, \ldots, n$, then $f \equiv 0$. Let us suppose we have such an $f$. 
Now writing $z=e^{i \theta}$ for $z$ on the unit circle $T=\{|z|=1\}$, we have

$$
z^{n} f(z)=P(z)=\sum_{k=0}^{2 n} b_{k} z^{k},
$$

where $P(z)$ is an algebraic polynomial of degree $2 n$ wose coefficient $b_{n}$ of degree $n$ satisfies $b_{n}=0$. Since the roots of $P$ are at the distinct points $e^{i \theta_{j}}$ and are all double roots, we see that $P$ is the square of a polynomial $Q$ of degree $n$ with $n$ roots on the unit circle. The next lema then settles the question.

LEMMA. Let $Q$ be a polynomial of degree with $n$ roots on the unit circle. Then the middle coefficient $b_{n}$ of $Q^{2}(z)$ does not vanish.

PROOF. Let

$$
Q(z)=\prod_{j=1}^{n}\left(z-e^{1 \theta_{j}}\right)
$$

so that

$$
\frac{Q^{2}(z)}{z^{n} \prod_{j=1}^{n}\left(-e^{i \theta_{j}}\right)}=\prod_{j=1}^{n}\left(z-e^{i \theta_{j}}\right) \prod_{j=1}^{n}\left(\frac{1}{z}-e^{-i \theta_{j}}\right) .
$$

Hence, on $\{|z|=1\}$, we have

$$
|Q(z)|^{2}=A \frac{Q^{2}(z)}{z^{n}},
$$

where

$$
A=\prod_{j=1}^{n}\left(-e^{-i \theta_{j}}\right) .
$$

Now integrate both sides of (3) around $T$ with respect to the measure $d \theta / 2 \pi=$ $(d z) /(2 \pi i z)$ to get 


$$
\mathrm{Ab}_{\mathrm{n}}=\int_{\mathrm{T}}|\mathrm{Q}(\mathrm{z})|^{2} \frac{\mathrm{dz}}{2 \pi i z}>0
$$

Since $|A|=1$, we see that $b_{n} \neq 0$.

ACKNOWLEDGEMENT. The research of the authors was partially supported by grants from the National Science Foundation, numbers NSF-550-34901 and NSF-MCS-78-01417 respectively.

\section{REFERENCES}

1. Davis, P. J. Interpolation and Approximation, New York, 1963.

2. Erdös, P. On the number of terms in the square of a polynomial, Nieuw. Arch. v. Wisk., 23(1948), 63-65.

3. Rényi, A. On the minimal number of terms of the square of a polynomial, Hungarica Acta Mathematica, 1(1947), 30-34.

4. Verdenius, w. On the number of terms of the square and the cube of polynomials, Indag. Math., 11(1949), 459-465. 


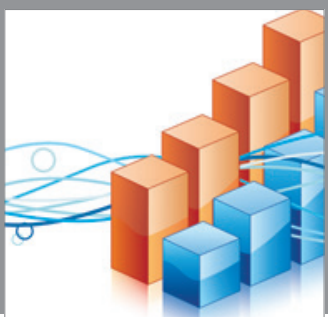

Advances in

Operations Research

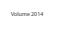

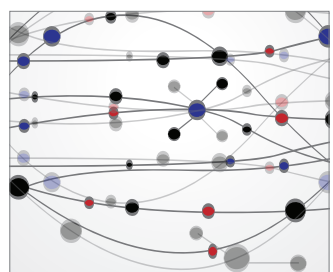

\section{The Scientific} World Journal
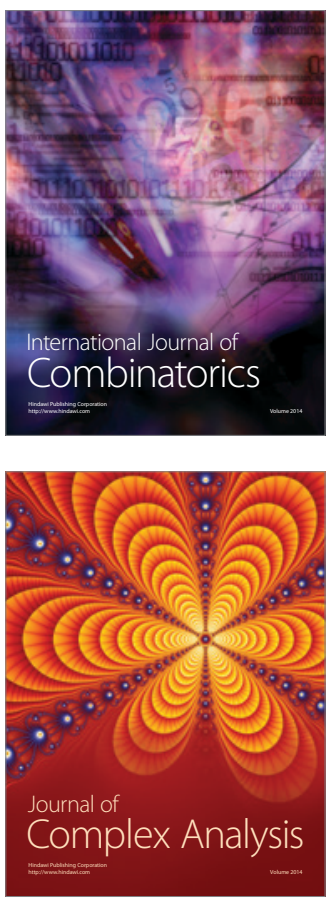

International Journal of

Mathematics and

Mathematical

Sciences
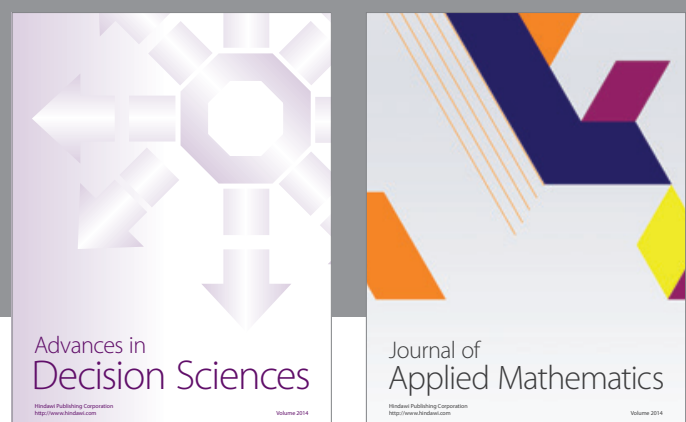

Journal of

Applied Mathematics
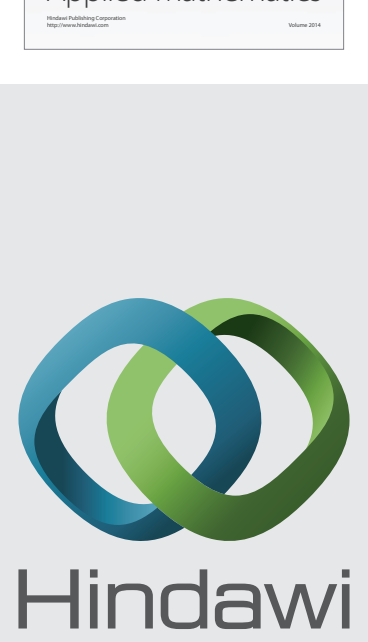

Submit your manuscripts at http://www.hindawi.com
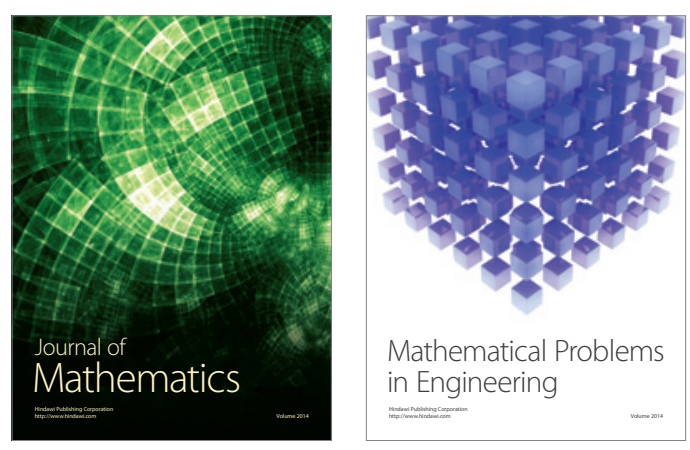

Mathematical Problems in Engineering
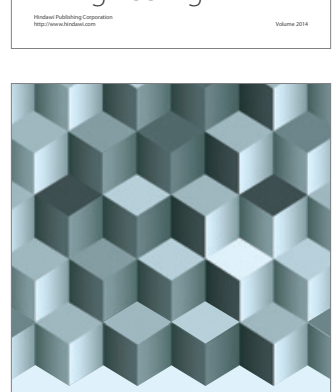

Journal of

Function Spaces
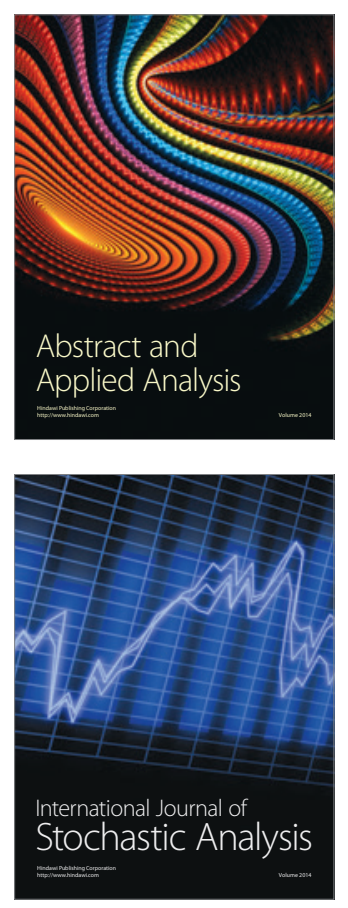

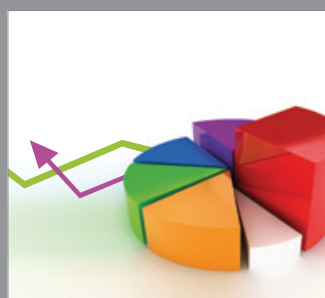

ournal of

Probability and Statistics

Promensencen
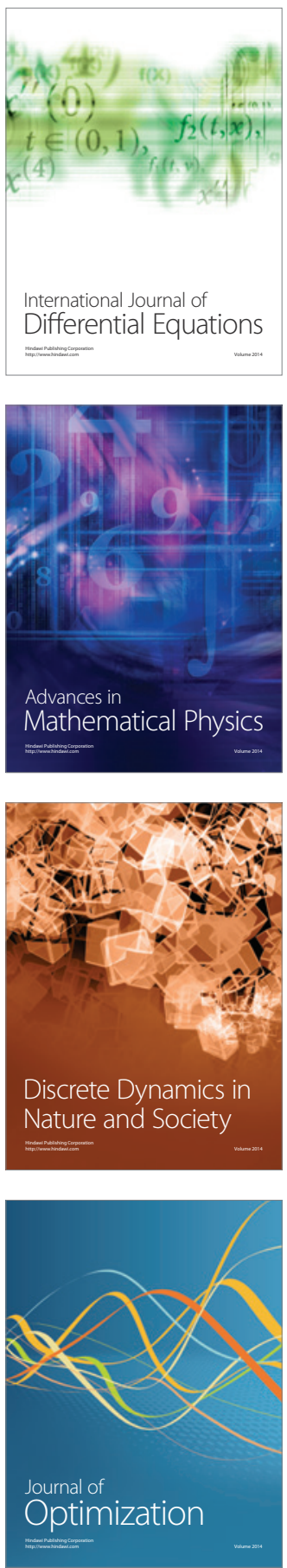